\title{
SEGMENT AND TRACK NEURONS IN 3D BY REPULSIVE SNAKE METHOD
}

\author{
Hongmin Cai ${ }^{1}$, Xiaoyin Xu ${ }^{2,3}, J u L^{4} u^{4}$ Jeff Lichtman ${ }^{4}$, S.P. Yung ${ }^{1}$ and Stephen T.C. Wong ${ }^{2,3}$ \\ ${ }^{1}$ University of Hong Kong, Department of Mathematics, Hong Kong, China. \\ ${ }^{2}$ Center for Bioinformatics, Harvard Center for Neurodegeneration and Repair \\ Harvard Medical School, Boston, MA, USA. \\ ${ }^{3}$ Department of Radiology, Brigham and Women's Hospital, Boston, MA, USA. \\ ${ }^{4}$ Harvard University, Department of Molecular and Cellular Biology, Cambridge, MA, USA.
}

\begin{abstract}
We present a snake (active contour) model based on repulsive force to segment neurons obtained from microscopy. Based on these segmentation results, we track the neurons in 3D image to look for its branch structure. These segmentation results allow user to study morphology of neurons to further investigate neuronal function and connectivity. This repulsive snake model can successfully segment two or multiple neurons that are close to each other by some alternating repulsive force generated from the neighboring objects. We apply our results on real data to demonstrate the performance of our method.
\end{abstract}

\section{INTRODUCTION}

To understand neuronal functions and connectivity of neurons it is important to study the morphology of neurons in three dimensions $[1,2]$. Neurons, such as motor neurons, transmit neuronal impulses from the central nervous system to a muscle or other effector tissue. The morphology of motor neurons including their branch structure and length are important to neuroscientists $[3,4]$. Neurons can be imaged under microscope after being stained by green fluorescent protein or obtained from transgenic mice [1]. Usually there are multiple neurons in a 3D image stack and each neuron need to be tracked in the $3 \mathrm{D}$ space to look for morphology.

In this paper we present a method based on a snake model to segment neurons consecutively on 2D image and then track these neurons in 3D images. In many cases, neurons are very close to each other and so the major task in our problem is to separate one neuron from its neighboring neurons as well as from its neighboring background. This task is very challenging because neurons may appear touching each other due to the low resolution and the orientation of the images. We extend the standard snake model to a repulsive force model to correctly track each neurons. Results obtained from real data show that the new method can successfully segment and track neurons.

H. Cai's work is done during a visit to the Center for Bioinformatics, Harvard Center for Neurodegeneration and Repair. This work was supported by a Center for Bioinformatics Program grant of the Harvard Center for Neurodegeneration and Repair, Harvard University, Boston, MA, USA.

\section{REPULSIVE SNAKE MODEL}

Snakes (active contour) are deformable curves that can move and change their shapes to conform to boundaries of objects in an image $[5,6]$. The movement and deformation are controlled by internal forces and external forces. The $2 \mathrm{D}$ parametric snake is to minimize the energy function $E(C)$ of a curve $C=(x(s), y(s))$ in image $I$ [5]. The snake is parameterized by $s \in[0,1]$ and the energy is given by

$$
E(C)=\int_{0}^{1}\left(\frac{1}{2}\left[\alpha\left|C^{\prime}(s)\right|^{2}+\beta\left|C^{\prime \prime}(s)\right|^{2}\right]\right) d s+\lambda f_{e x t}
$$

where $\alpha, \beta$ and $\lambda$ are positive coefficients, and $C^{\prime}$ and $C^{\prime \prime}$ denote the first and second derivative of $C$ with respect to $s$, respectively. $f_{\text {ext }}$ represents the external energy which generally takes the form of gradient magnitude, i.e., $f_{\text {ext }}=-\int_{0}^{1}|\nabla I(t)|^{2} d s$.

In practice, the minimization of $E(C)$ is performed iteratively by considering the curve as a function of an abstract time $t$, i.e., $C(s, t)$. Starting from an initial guess $C(s, t=0)=C_{0}$, the curve is deformed according to the following evolution equation until it reaches an equilibrium

$$
C_{t}=\alpha C^{\prime \prime}-\beta C^{\prime \prime \prime \prime}+\nabla f_{e x t}
$$

However, a snaked as defined above has its shortcomings, such as short capture range and limited deformability.

\subsection{GVF snake}

To overcome the problem of a standard snake, $\mathrm{Xu}$ and Prince [6] developed a new external force for parametric snake model. In the gradient vector flow (GVF) model [6], the external force in (1) is replaced by a new vector field $v$, which is a diffusion of the gradient vectors of a grey level or binary edge map derived from the original image. Variational minimization of such diffusion process results in the following two Euler equations

$$
\begin{aligned}
& \mu \nabla^{2} u-\left(f_{x}^{2}+f_{y}^{2}\right)\left(u-f_{x}\right)=0 \\
& \mu \nabla^{2} v-\left(f_{x}^{2}+f_{y}^{2}\right)\left(v-f_{y}\right)=0
\end{aligned}
$$

where $u, v$ is the "interpolated vector" and $f$ is the edge force $f=$ $|\nabla I|^{2}$. Because the gradient information from the strong object boundaries is propagated throughout the image by the diffusion process, the GVF model has a much larger "capture range" than 
the original snake model and is considerably less sensitive to initialization. This property allows it to track boundaries of objects with a much robust initialization $[7,8,9,10]$.

Although the GVF snake model has proven to outperform standard snake model in increasing capture range and deforming into concave boundaries [6], it may not detect weak boundaries. As pointed out in [7], the diffusion process (3) will progressively leads to strong boundary and overwhelms the force from weak boundaries. This defect leads snakes to deform into incorrect boundaries even when the initialization is positioned close to the true boundaries of objects. Another problem of GVF snake model is that it can not correctly segment objects that are adjacent to each other [11]. These two limitations greatly affect the application of snake in tracking problems, especially in our cases where neurons are likely to be close to each other. In some cases snake of one neuron evolves into the region of a neighboring neuron due to the weak boundaries between the two neurons.

\subsection{Repulsive GVF model}

Due to the limitation of resolution and imperfection staining process, neurons may appear touching each other. This makes it difficult to use the standard or GVF snake to segment neurons. To correctly separate touching neurons, we exploit the prior information that neurons are actually separated and employ a repulsive force to push the snakes toward their legitimate boundaries. This repulsive force can be obtained by reversing the gradient direction of neighboring objects.

We begin with the assumption that the domain of an image $u(x, y)$ consists of two objects that are close to each other, $O_{1}$ and $\mathrm{O}_{2}$, Fig. 1, and the rough area they belong to are known advanced. We want to determine an evolution that will continuously attract a robustly placed initial curve $\overrightarrow{C_{1}}$ toward the boundary of object $O_{1}$, and another $\overrightarrow{C_{2}}$ toward object $O_{2}$. We use $f_{e}$ to denote the edge forces of the trial image which includes objects $O_{1}, O_{2}$. We use the term influence parameters $I_{p 1}, I_{p 2}$ to represent the range parameters of the rough objects $O_{1}, O_{2}$ respectively. Such parameters can be the center and radius of a circle or vertex coordinates of a rectangle, which enclose our interested objects. Obviously, these parameters play an important role on the affectivity of our model. Fortunately, such parameters can be obtained from prior slice information in our neuron tracing problems.

The repulsive force for object $O_{1}$ can be formulated as

$$
f_{r 1}=\left\{\begin{array}{c}
f_{e}(I), I \in I_{p 1} \\
-f_{e}(I), I \ni I_{p 1}
\end{array}\right.
$$

where $f_{e}=-\nabla f$ is the inverted edge force. The repulsive force for object $\mathrm{O}_{2}$ can be defined similarly. To increase the capture range of snake we adopt the diffusion method (3) of [6]. This approach encourages snake to deform robustly in the right direction, even when initial contour is close to other objects. In fact this approach moves the "equilibrium line" to a much larger area, as indicated in Fig. 1. Similarly the repulsive procedure can be applied to another contacted subject in the same manner, for example in the segmenting of object $O_{2}$. With the same step we can have the repulsive force from $O_{1}$, and this repulsive force plus object boundary information will penalize the initialization curve, $\overrightarrow{C_{2}}$, to legitimate object $\mathrm{O}_{2}$.

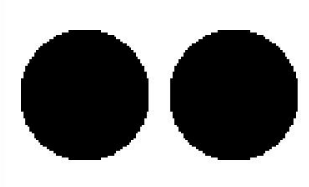

(a)

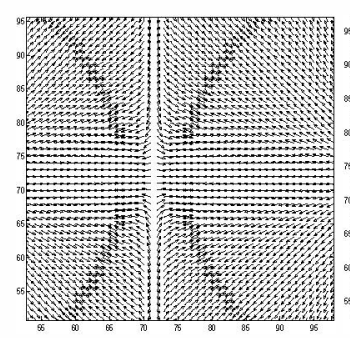

(b)

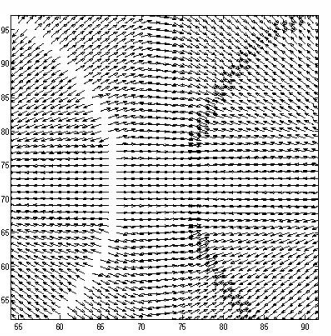

(c)
Fig. 1. Modified gradient direction and its intermediaries. (a) Synthetic image, object to the left is $O_{2}$ and to the right is $O_{1}$, (b) the associated gradient flow and the "equilibrium line" denotes the critical area of boundary influence. If we place the initial curve $\overrightarrow{C_{1}}$ across this line, the force will attract the curve to the wrong direction, (c) reversed gradient direction. The initial curve will deform to the right direction even if the initial curve is placed very close to the neighboring object.

\section{SEMI-AUTOMATIC NEURON SEGMENTATION}

In implementation, at first each 2D image is pre-processed by tophat and bottom-hat operations together with a median filtering to enhance image quality and remove shot noises. Next, to start the automatic segmentation and tracking process, we manually draw initial snakes on the first 2D image. Starting from the initialization, the repulsive snake method proceeds to the next image and segment neurons using the results from the previous $2 \mathrm{D}$ image as the initialization. Since there is little change of a neuron's shape and position between consecutive slices of 3D image, it is reasonable to use the final snakes of the previous slice as the initialization for the current slice, thus allowing an automatic initialization that link neurons positions across slices. However, we need a valid validation procedure to verify that the initialization contours are in the right position and snakes deformation are into the right boundaries, even when neurons are very close to each others. To address these two problems we use the radius and area of a neuron as a rule and adaptively adjust the repulsive force to control the deformation.

Because the center of mass of a snake does not change abruptly, we exploit this fact by revising the center of mass of a snake of slice $n+1$ by the recursive equation

$$
O_{c}=\frac{O_{e}}{n+1}+\frac{\bar{O}}{n+1} n
$$

where $O_{c}$ represents the revised center of mass, $O_{e}$ is the center of 
the current snake and $\bar{O}$ is the mean value of center of all previous slices, $n+1$ denotes the total of number of slices computed.

In addition, axon area will also change very little. Assume we have a well segmented contours $\overrightarrow{C_{1}}, \overrightarrow{C_{2}}$ for two touching cells $O_{1}, O_{2}$ in the $n$th slice $S_{n}$, we project the two contours to the next preprocessed slice $S_{n+1}$. We already know the areas and centers for contours $\overrightarrow{C_{1}}, \overrightarrow{C_{2}}$. Based on these prior knowledge we assign certain statistical "degree", denoted as $d(i), i=1,2$ being a convex function that reflects the degree of the realiability of the segmentation contours $\vec{C}_{i}$.

$$
d(i)=1-\gamma\left|\frac{A\left(C_{i}\right)-\bar{A}\left(C_{i}\right)}{\bar{A}\left(C_{i}\right)}\right|+(1-\gamma)\left|\frac{O_{C_{i}}-\bar{O}_{C_{i}}}{\bar{O}_{C_{i}}}\right|
$$

where $\gamma, 0<\gamma<1$ is a constant controlling the weight of the area and the changes in the center of mass, $\vec{A}(C)$ represents the mean area value of the current object in all slices, $A\left(C_{i}\right)$ denotes the area of current object $O_{i}$ enclosed by snake $C_{i}$. Similarly, we use $\bar{O}_{C_{i}}$ to denote the mean center of object $C_{i}$ and $O_{C_{i}}$ denote the center of mass of neuron $C_{i}$. Here $\gamma$ is set to 0.5 .

Such parameter can be employed to adjust the strength of repulsive force $f_{r}$ of (5), a revised repulsive force $f_{r}$ could be redefined as

$$
f_{r}=\left\{\begin{array}{c}
f_{e}(I), I \in I_{p} \\
-f_{e}(I) * d, I \ni I_{p}
\end{array}\right.
$$

The revised force will attain both robustness and adequacy. In fact, it is typical that two contacted objects with one has strong edge force and another shows weak edge force in their conjunction part. If we reverse the strong edge force, the energy force (2) will grow so high that it will push the snake apart from legitimate edge. Such problem is due to the fact that strong edge force will overwhelm the weak force during the diffusion process. We found that the adaptively revised force $f_{r^{\prime}}$ yielded much better results.

\section{RESULTS AND DISCUSSION}

We tested the repulsive snake method over a 3D image of size $512 \times 156 \times 512$. There are six neurons in the image. We use three cases to show the effectiveness of the new method in segmenting and tracking neurons, Fig. 2. We also compared the results of method with those obtained from GVF snake model [6]. From Fig. 2 we note that snake 1 and 2 deform into incorrect contour, Fig. 2(a). In order to correct this error we applied repulsive snake and obtained the correct results, Fig. 2(b). This adjustment keeps the snakes evolving to correct boundary, Fig. 2(c) and (d), until they yield incorrect results again, Fig. 2(e). Then based on the previous results and applying repulsive snake again, we obtain the correct segmentation results that place the snakes accurately along the boundaries of neuron 1 and 2, Fig. 2(f). In comparison, if we repeat the same initialization and use GVF snake, the obtained contours would very likely touch each other and yield less optimal results, as shown by the dotted lines in Fig. 2(a-f)

In summary the new method can segment multiple objects and does not increase computational complexity very much. The method can be used in other applications such cell tracking and blood vessel segmentation.

\section{CONCLUSIONS}

In this paper we developed a repulsive snake model to track and segment elongated objects in $3 \mathrm{D}$ images. The method processes
$2 \mathrm{D}$ images in sequence and uses the segmentation result of the previous image as the initialization contours for the next image. To overcome the problem that one snake may merge with one and other incorrectly, we introduced repulsive force to keep the two or multiple snakes separated. Application on real 3D microscopy images show that the method can yield robust and accurate results in segmenting neurons. The method can also be extended to other applications in segmentation, edge detection and tracking. Future work will focus on developing algorithms to model branches, i.e., split and merge, in axons. Performance can be enhanced by developing faster algorithms using the consideration that neurons do not undergo abrupt changes.

\section{REFERENCES}

[1] G. Feng, R.H. Mellor, M. Bernstein, C. Keller-Peck, Q.T. Nguyen, M. Wallace, J.M. Nerbonne, J.W. Lichtman, and J.R. Sanes, "Imaging neuronal subsets in transgenic mice expressing multiple spectral variants of GFP," Neuron, vol. 28, no. 1, pp. 41-51, Oct. 2000.

[2] K.M. Knobel, E.M. Jorgensen, and M.J. Bastiani, "Growth cones stall and collapse during axon outgrowth in Caenorhabditis elegans," Development, vol. 126, pp. 44894498, 1999.

[3] N. Kasthuri and J.W. Lichtman, "The role of neuronal identity in synaptic competition," Nature, vol. 424, no. 6947, pp. 426-430, 2003.

[4] C. Keller-Peck, M. Walsh, W. Gan, G. Feng, J. Sanes, and J.W. Lichtman, "Asynchronous synapse elimination in neonatal motor units studies using GFP transgenic mice," Neuron, vol. 31, no. 3, pp. 381-394, 2001.

[5] M. Kass, A. Witkin, and D. Terzopoulos, "Snakes: Active contour models," Int. J. Comput. Vis., vol. 1, pp. 321-332, 1987.

[6] C. Xu and J. L. Prince, "Snakes, shapes, and gradient vector flow," IEEE Trans. Image Proc., vol. 7, no. 3, pp. 359-369, 1998.

[7] C. Zimmer, E. Labruyere, V. Meas-Yedid, N. Guillen, and J.C.Z. Olivo-Marin, "Segmentation and tracking of migrating cells in videomicroscopy with parametric active contours: a tool for cell-based drug testing," IEEE Trans. Medical Imag., vol. 21, no. 10, pp. 1212-1221, May 2002.

[8] N. Ray, S.T. Acton, and K. Ley, "Tracking leukocytes in vivo with shape and size constrained active contours," IEEE Trans. Medical Imag., vol. 21, no. 10, pp. 1222-1235, Oct. 2002.

[9] N. Ray and S.T. Acton, "Motion gradient vector flow: an external force for tracking rolling leukocytes with shape and size constrained active contours," IEEE Trans. Medical Imag., vol. 23, no. 12, pp. 1466-1478, Dec. 2004.

[10] M. Jacob, T. Blu, and M. Unser, "Efficient energies and algorithms for parametric snakes," IEEE Trans. Medical Imag., vol. 13, pp. 1231-1244, 2004.

[11] X. Xie and M. Mirmehdi, "PAGS: region-aided geometric snake," IEEE Trans. Image Proc., vol. 13, no. 5, pp. 640652, May 2004. 


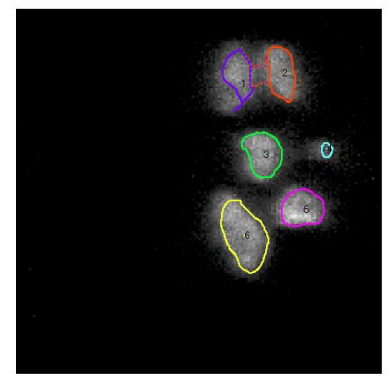

(a)

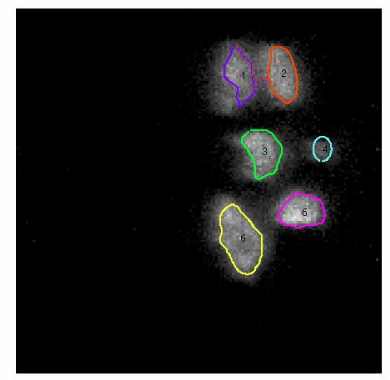

(c)

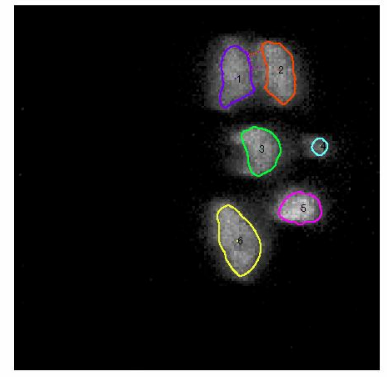

(e)

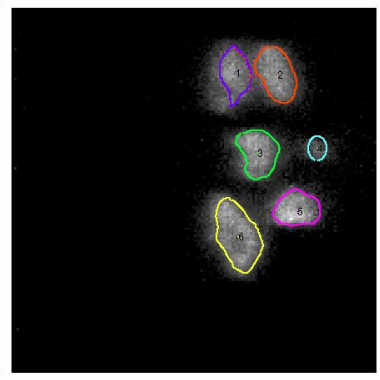

(b)

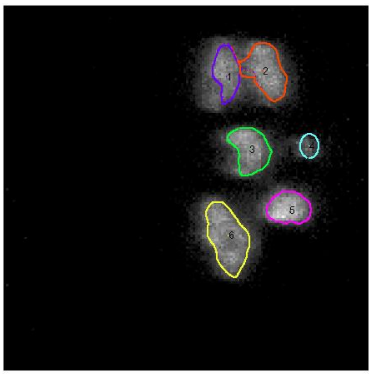

(d)

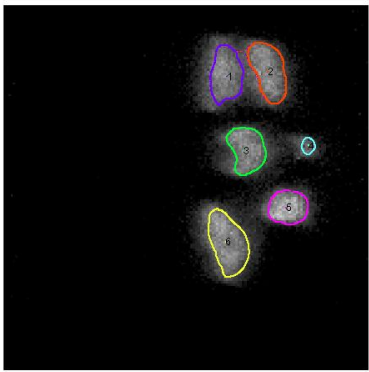

(f)

Fig. 2. Neuron tracking and segmentation on the six slices using GVF snakes and repulsive GVF snakes. Solid lines show the evolution result of repulsive GVF snakes and dotted lines are results of GVF snakes. (a-f) Evolution results show incorrect intermediate results and the correct results obtained by repulsive GVF snake. 